\title{
Optimizing logistic regression coefficients for discrimination and calibration using estimation of distribution algorithms
}

\author{
V. Robles - C. Bielza - P. I arrañaga - S. González • \\ L. Ohno-Machado
}

\begin{abstract}
I ogislic regression is a simple and efficient. supervised learning algorilbm for estimating the probability of an outcome or class variable. In spite of its simplicity, logistic regression has shown very good performance in a range of fields. It is widely accepted it a ratige of liclds because its results are casy to interpret. Fitling the logistic regression model usually involves using the principle of maximum likelihood. The Newton-Raphson algorithm is the most common numerical approach for obtaining the coefficients maximizing the likelihood of the data.

This work presents a novel approach for filling the logislic regression mode] based on estimation of distribution algorithms (EDAs), a tool for evolutionary computation. EDAs are suitable not only for maximizing the likelihood, but also for maximizing the area under the receiver operating characteristic curve (AIC).

Thus, we tackle the logislic regression problem from a double perspective: likelihood-based to calibrate the model and $A U C$-based to discriminale between the different classes. Under these two objectives of calibration and discrimination, the Pareto front can he obtained in our LDA framework. These fronts are compared with those yielded by a multiobjeclive $\mathrm{ED} \wedge$ recently introduced in the literature.
\end{abstract}

Keywords Logistic regression - Lvolutionary algorithms - Lstimation of distribulion algorilhms - Calibration and discrimination

Mathematics Subject Classification (2000) 62J12 - 90C59.90C29 


\section{Introduction}

Logistic regression modeling is employed in many fields (Hosmer and Lemeshow 2000). The outcome variable is binary, while the explanatory variables are of any lype, lending great flexibility to this approach. Fxperimental results have shown that logistic regression can perform at least as well as a more complex classifier in a variety of data sets (Baumgartner et al. 2004; Kiang 2003), and this approach compared favorably with many supervised machine learning techniques: $k$-nearest neighbors, discriminant. analysis, neural networks, support vector machines, and decision trees.

As in other simpler regression models, logistic regression applies the maximum likelihoof principle for parameter estimation. The model that linearly relates the log of the odds of the response and the explatalory variables gives rise lo complex nonlitsear likelihood equations in the unknown parameters (Ryan 1997). Therefore, special numerical methods for their solution are required.

The so-calted Newton-Raphson method is commonly used to solve the likelihood cqualions numerically. Nlthough the method requires inverting a malrix and exhibits some dependence on the initial starting conditions for convergence to be guaranteed, it shows good performance overall (Minka 2003). One of the aims of the current paper is to lackle this maximizalion problem for parameler estimalion by using a recent. optimization heuristic called estimation of distribution algorithms (LDAs) (Larrañaga and Lozano 2002). In this sense, we contribute towards the (currently poor) applicalion of optimizalion heuristics in stalistic estimalion and modelling problems (Winker and Gilli 2004).

LDAs are evolutionary algorithms that are among the best-known stochastic population-based search methods. These algorithms construct an explicit probability model from a set of selected solutions which is then conveniently used to generate new promising solutions in the next iteration of the evolutionary process. Other evolutionary algorilhms, like genelic algorilhms, have been used in logislic regression but for performing feature subset selection (Vinterbo and Ohno-Machado 1999a; Nakamichi et al. 2004), not for estimating the parameters or investigating the model performance from several points of view. To our knowledge, this is the first exploration on how FDAs can be used in this contexl.

The search for the parameters, both in a numerical or in an evolutionary way, tries to attain an appropriate model in the sense of maximizing the chances of obtaining the data given the filled model. The proximily of the true and the observed probability for a given set of observations, usually measured using calibration indices, is an important criterion of a model performance. Iowever, it does not suffice. The logistic regression model outpuls the probability of a cerlain event occurring. This probability can be used to predict the class. Ior classification, high discriminatory ability to differentiate between the classes is at least as important as calibration. In fact, the recommendation of assessing the model performance by considering both calibralion and discrimtination has ben elearly asserict, c.g., in seminal texis on logistic regression (see Hosmer and Lemeshow 2000, p.163).

Since good calibration does not necessarily mean good discrimination and vice versa, both lypes of measures should be analyzed in logistic regression models. Therefore, we specifically study the behavior of two model performance measures: 
the maximum (log) likelihood (for calibration) and the area under the receiver operating characteristic curve (AUC) (for discrimination).

Among the most outstanding strengths, our new LDA framework can be flexible cnough to cope with the parameter estimation when the optimization is bascd on calibralion or on tiscrimination, or even on olher mole] per [ormance measures like the Brice score or aty mulit-objeclive measure. Moreover, the hi-objeclive space of caljbration against discrimination can be explored to depict the relationship between both objectives, allowing us to estimate the Pareto front with the non-dominated points.

The paper is organized as follows. Section 2 reviews the logistic regression model and the derivation of the likelihood equations. Section 3 includes the model performantce measures we will use lo assess calibration and discriminalion. Seclion 4 deals with diferent cstimalion methods of performance when the model faces luture (uj]seen) data. Section 5 describes a method for searching the logistic regression parameters based on EDAs, emphasizing the advantages of this new approach. Section 6 shows the experiments with several data sets and the potentiality of our method. Section 7 highlights the benefits of our new approach. I'inally, we discuss in Sect. 8 the conclusions and lines of lul.ure rescarch.

\section{Logistic regression}

Logistic regression (Hosmer and Lemeshow 2000) is a standard method to describe the relationship hetween a response (or dependent) variable which is hinary and several explanatory (or predictor) variables called covariates. When it is used for classification purposes, the response variable is the class variable $C$ predicted through covariales $X_{1}, \ldots, X_{k}$. In this contexl, logistic regression becomes a powerful supervised classificalion praradigm that provides explicil probabilities of classificalion that. can be used to provide class label information. This approach falls into the category of discriminative clatssifiers, in the sense that they model the probability of the class given the covariates, in contrast to generative classifiers that model the joint probability of the class and the covariates ( $\mathrm{Ng}$ and Jordan 2001). As opposed to other methods like discriminant analysis, strong assumplions like normal distribulion of the covariales giver the class are not required. Also, covariales can be given in a quantitalive (continuous or discrete) or qualitative scale.

The logistic regression classifier is induced from a (training) data set $D_{N}$ containing $N$ independent samples $\mathcal{D}_{N}=\left\{\left(c_{j}, x_{j 1}, \ldots, x_{j k}\right), j=1, \ldots, N\right\}$, drawn from the joint probability distribution on $\left(C, X_{1}, \ldots, X_{k}\right)$. In this paper, we focus on the two category classification problem, although the ideas conld be readily extended to the multi-calcgory case. Thus, $C$ can only lake 0 and 1 values, where label $c_{j}=1$ means that the $j$ th inpul pallern $\mathbf{x}_{j}=\left(x_{j 1}, \ldots, x_{j k}\right)$ is in the first class (i.c., obscrvalion $j$ has the feature that $C$ represents), while $c_{j}=0$ means $x_{j}$ does not have the feature, and therefore belongs to the other class. The classification model will be used for assigning labels $c_{j}$ to new instances that are not part of the training set, and therefore are only characterized with the values of the predictor variables. 
Let $\pi_{\mathbf{x}}$ denote $P(C=1 \mid \mathbf{x})=P\left(C=1 \mid X_{1}=x_{1}, \ldots, X_{k}=x_{k}\right)$. Then the logit model is defined as:

$$
\log \frac{\pi_{\mathbf{x}}}{1-\pi_{\mathbf{x}}}=\beta_{0}+\beta_{1} x_{1}+\cdots+\beta_{k} x_{k}
$$

or equivalently,

$$
\pi_{\mathbf{x}}=\frac{\mathrm{e}^{\left(\beta_{0}+\beta_{\left.1 \cdot x_{1}+\cdots+\beta_{k} x_{k}\right)}\right.}}{1+\mathrm{c}^{\left(\beta_{0}+\beta_{1} x_{1}+\cdots+\beta_{k} x_{k}\right)}}=\frac{1}{1+\mathrm{e}^{-\left(\beta_{0}+\beta_{1} x_{1}+\cdots+\beta_{k} x_{k}\right)}},
$$

where $\boldsymbol{\beta}=\left(\beta_{0}, \beta_{\mathrm{I}}, \ldots, \beta_{k}\right)$ denotes the vector of regression coefficients including a constant or intercept $\beta_{0}$.

Therefore, the model specifies $\pi_{\mathrm{x}}$ as the dependent vatiable to be a function of the predictor variables. Since $C$ is dichotomous, its expected value is $E(C \mid \mathbf{x})=\pi_{\mathbf{x}}$, and we search for a relationship hetween the expected response and the covariates.

Regression coeflicients are usually estimaled from the dala by meats of the maximum likelihood estimation method. Given the training data set, the likelihood function is $\mathcal{C}(\boldsymbol{\beta})=\prod_{j=1}^{N} \pi_{\mathbf{x}_{j}}^{c_{j}}\left(\mathbf{1}-\pi_{\mathbf{x}_{j}}\right)^{1-c_{j}}$, where $\pi_{\mathbf{x}_{j}}$ is stated in (2). Maximum likelihood estimalors (ML L) $\hat{\beta}_{i}$ are obtained by maximizing $\mathcal{L}$ wilh respect to $\beta$, or equivalently, by maximizing log $\mathcal{L}$ with respect to $\beta$.

We have that

$$
\begin{aligned}
\log \mathcal{L}(\boldsymbol{\beta}) & =\sum_{j=1}^{N}\left(c_{j} \log \pi_{\mathbf{x}_{j}}+\left(1-c_{j}\right) \log \left(1-\pi_{\mathbf{x}_{j}}\right)\right) \\
& =\sum_{j=1}^{N} c_{j} \log \frac{\pi_{\mathbf{x}_{j}}}{1-\pi_{\mathbf{x}_{j}}}+\sum_{j=1}^{N} \log \left(1-\pi_{\mathbf{x}_{j}}\right)
\end{aligned}
$$

and using (1) and (2),

$$
\log \mathcal{C}(\beta)=\sum_{j=1}^{N} c_{j}\left(\beta_{0}+\beta_{1} x_{j 1}+\cdots+\beta_{k} x_{j k}\right)-\sum_{j=1}^{N} \log \left(1+\mathrm{e}^{\left(\beta_{0}+\beta_{1} x_{j 1}+\cdots+\beta_{k} x_{j k}\right)}\right) .
$$

Thus, the following system of $k+1$ equations and $k+1$ parameters-called the likelihood equationts - has to be solved:

$$
\begin{aligned}
\frac{\partial \log C(\boldsymbol{\beta})}{\partial \beta_{0}} & =\sum_{j=1}^{N} c_{j}-\sum_{j=1}^{N} \frac{\mathrm{e}^{\left(\beta_{0}+\beta_{1} x_{j 1}+\cdots+\beta_{k} x_{j k}\right)}}{1+\mathrm{e}^{\left(\beta_{0}+\beta_{1} x_{j 1}+\cdots+\beta_{k} x_{j k}\right)}}=0, \\
& \vdots \\
\frac{\partial \log \mathcal{C}(\boldsymbol{\beta})}{\partial \beta_{k}} & =\sum_{j=1}^{N} c_{j} x_{j k}-\sum_{j=1}^{N} x_{j k} \frac{\mathrm{e}^{\left(\beta_{0}+\cdots+\beta_{k} x_{j k}\right)}}{1+\mathrm{e}^{\left(\beta_{0}+\cdots+\beta_{k} x_{j k}\right)}}=0 .
\end{aligned}
$$

Unfortunately, there is no analytical solution of these nonlinear equations for $\widehat{\beta}_{i}$, but we may resort to using numerical optimization methods. Among these, a general 
choice is the Newton-Raphson numerical procedure (Thisted 1988) in which each iteration provides an updating formula given by

$$
\widehat{\boldsymbol{\beta}}^{\text {new }}=\widehat{\boldsymbol{\beta}}^{\text {old }}+\left(\mathbf{X}^{\mathbf{l}} \mathbf{W} \mathbf{X}\right)^{-1} \mathbf{X}^{\mathbf{l}}(\mathbf{c}-\widehat{\boldsymbol{\pi}}),
$$

where $\hat{\boldsymbol{\beta}}=\left(\hat{\beta}_{0}, \hat{\beta}_{1}, \ldots, \hat{\beta}_{k}\right)$, c denotes the vector of response values $c_{j}(j=$ $1, \ldots, N), \mathbf{X}$ denotes an $N \times(k+1)$ matrix with each row given by $\left(1, \mathbf{x}_{j}\right)$, $\hat{\boldsymbol{\pi}}$ denotes the veetor of estimated values al that iteralion, i.c., its jth-component. is $\pi_{\mathbf{x}_{j}}=\left[1+\mathrm{e}^{-\left(\beta_{0}^{\text {old }}+\hat{\beta}_{l}^{\text {old }} x_{j 1}+\cdots+\hat{\beta}_{k}^{\text {old }} x_{j k}\right)}\right]^{-1}, j=1, \ldots N$, and $\mathbf{W}$ denotes a diagothal malrix with elements $\hat{\pi}_{\mathbf{x}_{j}}\left(1-\hat{\pi}_{\mathbf{x}_{j}}\right)$. This formula is ased unli] a convergence criterion is achieved. Common convergence criteria consist of the detection of negligible changes in the log likelihood function, in the parameter estimates, or in the predictions. No single criterion appears superior to the others. in regard to starting estimates, the ones obtained using discriminant analysis turn out to be good and may speed up the convergence (Ryan 1997).

Minka (2003) compares eight different. numerical algorilhms for compuling the MLEs in terms of computational complexity (total floating-point operations) and performance (log likelihood value achieved). The Newton-Raphson algorithm shows excellent pertormance and a rapid convergence rate.

Therefore, il. may secm hard to design a better algorithm to approximate the $\widehat{\beta}_{i}$ MI.Fs lor logistic regression. Since our estimation problem is an oplimizalion problem, a promising alternative would be to try some optimization heuristics, which surprisingly have not been very commonly used in statistical estimation and modelling problems (Winker and Gilli 2004). We introduce here the estimation of distribution algorithms (LDAs) that, to the best of our knowledge, have never been used in this conlexi. $\Lambda$ s far as cvolutionary algorilhms are concerned, we only know of a genetic algorthm employed to select variables in logistic regression (Vinterbo and OhnoMachado 1999a; Nakamichi el al. 2004), not in the cslimation problem.

The $\log \mathcal{L}$ function guides the search of $\widehat{\beta_{i}}$ "s, trying to produce a model that fits, i.e., the observed sample values of the response variable agree with the values predicted by the model (or fitted values). This goodness-of-fit informs us about the effectiveness of the model in describing the response variable. A good fit provides a calibraled model.

However, when classification is a goal of the modeling and eslimated probabilities are used to predict the class membership, the discrimination between the different classes may not be accurate even if the model fits the data well. Situations in which the logistic regression fits the data properly but yields poor classification have been reporled clsewhere (sec Hosmer and I cmeshow 2000, p. 156 and p. 163).

Therefore, both calibration and discrimination measures should be analyred in logistic regression models. Among other interesting findings, we show that EDAs are flexible enotgh to cope with the estimation of $\beta$ 's when the optimization is based on any of those measures.

\section{AUC as a model perfornance measure in loggistic regression}

As noted above, good calibration does not necessarily entail good discrimination and vice versa. A seminal text on logistic regression claims that "model performance 
should be assessed by considering both calibration and discrimination" (see Itosmer and $I$ concshow 2000, p. 163 ) and this is also out vicwpoint.

One solution taken in the literature is to start off with a model that has gond discrimination and then adjust its calibration, what is called model recalibration Harrell et al. (1984). In logistic regression, IIarrell et al. (1996) propose shrinkage to recalibrate. However, this increases calibration only when the lestitg sel is relatively large (Steyerberg et al. 2004), which is not always the case in practice. Vinterbo and Ohno-Machado (1999b) propose to alter the estimated probabilities by applying a discrimination-preserving transformation, which is empirically determined from a linear regression on the points in the calibration plot to move them closer to the identity line (the ideal calibration). A second transformation is still needed to keep the resulting predictions within 0 and 1 . This method suffers from a number of limitations: It is not useful when the model gives the same estimates for every injul., when the calibration plot points are spread in an alternating pattern around the identity line, or when many estimates are close to either 0 or 1.

The approach taken in this paper is different, since we search for a model that directly provides good overall performanec by oplimizing with respect. to different. performance measures with an evolutionary algorithm.

When classifying instances, since probabilistic model outputs are continuous, we have to transform those outputs into binary outcomes providing the predicted class. For a given cutpoinl or threshold $t \in \mid 0$, I , we can decide that the prodicted class of an instance $j$ is $\hat{c}_{j}=1$ if its estimated probability $\hat{\pi}_{j} \geq t$ and it is $\hat{c}_{j}=0$ otherwise, Each $t$ results in a sensitivity and specificity pair. The plot of the values of 1-specificity (false positive rale) against. setısilivily (Iruc posilive ralc) over all possible cutpoints is the receiver operating characteristic (ROC) curve (see e.g. Pepe 2003).

ROC curves describe the predictive behavior of a classifier independently of elass distributions or error costs. Its use as a metric for comparing algorithms rather than using the classification error rate has been widely justified (see, e.g., Provost et al. 1998).

The ROC. curve is commonly summarized by the area under the curve (AUC) (Hilden 1991; Bradley 1997). AUC ranges from 0 to 1, where perfect discrimination between both clisses corresponds to an area of 1 (a horizontal line through the point $(1,1)$ ) and randon classification corresponds to an area of 0.5 (the identity line).

There are many ways to compute the AUC (lawcett 2003). We can use the trapezoidal rule atfer connecting the ROC curve points by straight lines (IIorton et al. 2004), which is a poor computational strategy, known to underestimate the AUC if the number of poitts is limited (Hanley and McNeil 1982). NUC. has an imporlant. statistical property: it is equivalent to the probability that the classifier will rank a randomly chosen positive instance higher than at randomly chosen negative instance. Thus, an intultive way to proced is by compuling the concordance inder or c-inder, as follows. Icl. us create all the possible pairs of observations such that its first element has $c_{j}=1$ and the second element has $c_{j}=0$. Then, the $c$-index is the proportion of the time that the observation with $c_{j}=1$ has the higher of the Iwo probabilitics, wilh lics resolved by lossing an unbiasod coin (Harrell ct al. 1996; Hanley and McNeil 1982). Without ties, the $c$-index is the AIC and is equivalent to 
the Mann-Whitney $U$ statistic, which is another form of the Wilcoxon rank-sum test (Hajek ol al. 1999). Under limiled informalion-like baving only a single point of the ROC curve-approximations to the AUC can be computed (van den Hout 2003 ). Other approximations, both parametric and non-parametric, have been proposed in the latge literature on the subject, sec a review in Jasko el. al. (2005).

\section{Model performance assessment}

In the previous sections, we have introduced two performance measures of a model: $\log \mathcal{C}$ and $\mathrm{AUC}$. To assess this performance that allows us to compare a model to olher candidate models, ils discrimination/calibralion ability has to be checked on test. data that is different from training data. By so doing, we estimate the generalization performance of our model.

I.el. us starl. our discussion by taking the error rale as the performance metric. After designing otr logistic regression classifier, its (classification) error rate when using the model for classifying unseen (new) instances has to be estimated, or at least its expected etror rale. $A$ low error rate usually corresponds to high accuracy. When comparing error estimators, that should be as close as possible to the true error, one has to consider their bias and variance, since the composition of both defines the mean squared crror. Unbiasedness (or al. Jeast a low degree ol bias) and small variance are desirable. A large variance is of particular concern even with unbiasedness, since the estimate corresponding to a given sample can be often far from the actual error rate. Anong these estimalors, the resubstitution estimator, where the error is directly computed on the sample data itself, is simply a very fast but usually optimistic (i.e. low-biased) estimalor of the Irue crror. Holdout crror estimalion, wilh a lraining set for the modeling and a test set for testing the classifier, requires large sample sizes.

I Iowever, cross-validation error estimation is the most widely used method and provides a nearly unbiased estimale of the fulure crror rate al hough perhaps at the expense of some variance. In $k$-fold cross-validation (Stone 1974), the data set is randomly partitioned into $k$ folds of approximately equal size. Each time $t \in[1,2, \ldots, k\}$ a fold is left out of the modeling process and used as a lesting sel. The cross-validalion estimate of the error is computed by averaging the resulting error estimations from all folds. A 10 -fold cross-validation will be the chosen method in this paper.

Any - more geteral - preromance measures as $\log L$ and $\Lambda U C$ may be eslimated from the data in a way similar to the one used in estimating the error rate.

\section{Estimating the logistic regression coefficients with estimation of distribution algorithms}

As stated in Secl. 2, the likelihood equations to be solved in order lo oblain the va]ues of parameters $\beta_{0}, \ldots, \beta_{k}$ cannot be resolved analytically (Hosmer and Lemeshow 2000). Several mumerical algorithms for computing the MLE of the regression coefficients have been proposed in the lilerature (Minka 2003). However, the solutions provided by these procedures are likely to be improved in some circumstances. 
In this section we present an introduction to estimation of distribution algorithms (FDAs), a rccent. population-based stochastic optimization heuristic (J arrafiaga and Lozano 2002). We also describe a method for searching the optimal values of the logistic regression cocficicnts based on EDAs in contituous domains.

\subsection{Fslimation of distribulion algorithms}

It is possible to use oplimization heuristics as an alternative way for the estimalion of regression parameters. These optimization heuristics can be divided into local and population-based search methods. Evolutionary algorilhms are among the best-known stochastic population-based search methods. They start from a random population of individuals - cach of them representing a possible solution to the optimization problem-and iterate until some pre-defined stopping criterion is satisfied. At every itcration, usually called generation, a subse of individuals is selected. By applying some variation operators to the selected set, a new population is created. An example of cvolutionary algorithms are genetic algorthms (GAs) (Goldherg 1989). The distinguishing feature of GAs is the application of the recombination and mutation operators. As mentioned before, GAs have been used in combination with logistic tegression only in the selection of the covariates to be included in the model (Vinterbo and Ohno-Machado 1999a; Nakamichi et al. 2004).

Another class of population-based search methods comprises those algorithms that use probabilistic modeling of the solutions instead of genetic operators. Estimation of distribution algorithms (Larrañaga and Lozano 2002; Lozano et al. 2006) are evolutionary algorithms that construct an explicit probability model from a set of selected solutions. This model can capture, by means of probabilistic dependencies, relevant interactions among the variables of the problem. The model can be conveniently used to generate new promising solutions.

Figure 1 shows a pseudo-code for a general FDA approach to oplimization, $\Lambda 1$ the beginning $M$ individuals, each of them representing a point of the search space, are gencraled at random. These $M$ individuals constilute the inilial population and are evaluated by means of a fitness function. In a first step of the algorithm, a number $N(N<M)$ of individuals are selected according to a selection method. Next, the

(i) $D_{0} \leftarrow$ Generale $M$ individuals randomly

(ii) $l=1$

(iii) do (

(iv) $\quad D_{l-1}^{S e} \leftarrow$ Select $N<M$ individuals from $D_{l-1}$ according to a setection method

(v) $\quad p_{l}(z)=p\left(z \mid D_{l-1}^{S e}\right) \leftarrow$ Estimate the joint probability distribution from the selected individuals

(vi) $\quad D_{l} \leftarrow$ Sample $M$ individuals (the new population) from $p_{l}(z)$

(vii) until a stopping critcrion is mel

Fig. 1 Psendo-code for the EDA approach to optimization 
induction of a multidimensional probabilistic model that reflects the interdependencies between the variables in these $N$ individuals is carried out. The estimation of the joint density constilutes the bot.lencek of EDAs, as different degrees ol dependencies between the variables used to represent the individuals can he considered. In a third step, $M$ new individuals the new population - are obtained from a simulation of the multidimensional probabilistic model learnt in the second step. These three steps are repeated until a stopping condition is met.

The main advantages of EDAs as compated to GAs are: (i) they avoid designing ad hoc crossover and mutation operators, as well as the tuning of the values of several associated parameters; (ii) they are able to express in an explicit manner, by means of a joint probability distribution, the relationships between the different variables used to represent a point of the search space, and (iii) they can incorporate from the beginning some knowledge we cant have about the problem by imposing condilional independence relationships between those variables. These advantages, as well as the difficulties of using real coded GAs, necessary in our problem, have led us to choose FD $\Lambda$ s as betcer suited for this paper.

LDAs have been successtully applied in machine learning, for instance in learning Bayesian networks from dala (Blanco el al. 2003; Romero et al. 2004), in fealure subset selection (Inza et al. 2000), and in different optimization problems, within knearest neighbors, clustering and neural networks paradigms (Larrañaga and Lozano 2002).

\section{$5.2 \mathrm{UMDA}_{c}^{G}$ approact for logistic regression}

Let $Z_{i}$, with $i=0,1, \ldots, k$, represent a continuous random variable. A possible value of $Z_{i}$ is denoted $z_{i}$. Each conlinuous variable is asscciated wilh its corresponding parameter of the togistic regression model. In our case, $z_{i}$ represents a value for parameter $\beta_{i}$. Similarly, we use $Z=\left(Z_{0}, Z_{1}, \ldots, Z_{k}\right)$ to represent a $k+1$ dimensional random variable and $z=\left(z_{0}, z_{1}, \ldots, z_{k}\right)$ to denote one of its possible values. In this sense, $z=\left(z_{0}, z_{1}, \ldots, z_{k}\right)$ refers to a value for the parameters $\beta=\left(\beta_{0}, \beta_{1}, \ldots, \beta_{k}\right)$. The joint density function over $Z$ is denoted by $p(z)$.

In order to reduce as much as possible the computational cost derived from the learning of the joint density function, $p(z)$, we have chosen the EDA approach called $U_{M A}^{G}$ (Larrañaga et al. 2000). UMDA $G$ assumes that at each generation all varjables are independent and normally distributed. Tacking these two assumptions into account, the joint density at each generation, $p_{l}(z)$, can be factorized as follows:

$$
p_{l}(z)=\prod_{i=0}^{k} p_{l}\left(z_{i}\right)=\prod_{i=0}^{k} \frac{1}{\sqrt{2 \pi} \sigma_{i l}} \mathrm{e}^{-\frac{1}{2}\left(\frac{z_{i}-\mu_{i l}}{\left.\mathrm{o}_{i l}\right)^{2}}\right.} .
$$

The $2(k+1)$ parameters of the model, $\mu_{i l}$ and $\sigma_{i l}$ with $i=0,1, \ldots, k$, have to be estimated at eacl generation by means of the sample mean and standard deviation calculated from the selected individuals.

We can use libAs not only to obtain the values of the parameters $\beta_{01}, \beta_{1}, \ldots, \beta_{k}$ that maximize the likelihood bul. also to optimize other model performance moasures like the AUC. We propose the use of EDAs, specifically the UMDA ${ }_{i}^{G}$ approach, to build two new algorithms that use different fitness functions: 
- UMDA ${ }_{c}^{G}-\log L$ which goal is to obtain the $\beta$ 's in a logistic regression model, with the highest $\log f$ value;

- UMD ${ }_{c}^{G}-\Lambda U C$, which goal is 10 oblain the $\beta$ 's in a logislic regression model, wilh the highes!. $\Lambda U C$. value.

Note that, unlike the traditional procedures to find parameters $\beta_{i}{ }^{7} \mathrm{~s}$ as MT.F, the EDA apjoroach is able to use any oplimization objective, regardless of ils complexity or the lack of an explicit formula for its expression, like for the AUC objective.

The parameters used to run the proposed algorithms based on LDAs and the method used for assessing the convergence may vary depending on the specific problem. The chosen values for our data sets are fully detailed in Sect. 6.2. As usual, the best individual in the last. generalion is chosen as solution.

Other EDA approaches thal. lake into accouml more complex interactions among parameters $\beta_{j}$ 's could be used, at the expense of the computational cost, but with the explicit modeling of their probabilistic conditional dependencies (see Larrafiaga and Lozano 2002, Chap. 2).

\section{Experiments}

\subsection{Dala sels}

The experimental study was carried out. wilh six diflerent. dala sets, described as collows:

- The Breast cancer data set intends to distinguish benign tumors from malignant tumors (benign/malignant).

- The Diabetes data set shows whether the patients have signs of diabetes (healthy/diabeles) according lo World Healh Organization crileria.

- The ICU data sel contains patients who were part of a much larger study on survival of patients following admission to an adult intensive care unit (ICU). The major goal of this study is to develop a logistic regression model (or another valid method) to predict the probability of in-hospital survival (died/lived).

- The Prostate cancer data set involves a study of patients with prostate cancer. The goal of the analysis is to determine if variables measured at a baseline exam cat be used to predicl whether the tumor has penetrated (penctrated/nol penetrated) the prostalic capsule.

- The purpose of the UIS data sel is to compare Ircalment programs of different. planned durations designed to reduce drug abuse and to prevent high-risk HIV behavior. The class variable is defined as having returned to drug use prior to the scheduled completion of the treatment program (remained drug free/ otherwise).

- The goal of the Adult-r data set is to predict whether income exceeds $\$ 50$ $\mathrm{K} / \mathrm{yr}(>50 \mathrm{~K}, \leq 50 \mathrm{~K}$ ) based on census data. The original Adult data set includes 48,842 inslances. By randomly selecting 8,000 instances maintainting the same proportion of posilive and regalive cases, the corresponding reduced version Adult-r is the data set used here. 
Tahle I Data sets characteristics

\begin{tabular}{lccc}
\hline Data set & Variables & Instances & Positive instances \\
\hline Breast & 10 & 699 & 458 \\
Diabetes & 9 & 768 & 268 \\
ICU & 20 & 200 & 40 \\
Prostate & 8 & 380 & 153 \\
UIS & 8 & 575 & 147 \\
Adult-r & 14 & 8,000 & 1,912 \\
\hline
\end{tabular}

The first two and the last data sets come from the UCI machine learting repository (Newman et al, 1998). The remaining data sets were obtained from (10smer and Lemeshow 2000). 'lable 1 contains the number of variables, the number of instances and the number of positive instanes $\left(c_{j}=1\right)$ of each data set.

\subsection{Implemenl.alion}

To obtain the MLEs of $\beta$ 's with the Newton-Raphson algorithm, we have used the $R$ cuvironment for slatislical compuling and graphics (R Developmetil Core Team 2004; Thaka and Gentleman 1996), which is freely available. MLEs are computed using the $g \ln$ () function that lakes into account the change between successive steps in parameter estimates to assess the convergence of the algorithm. We have called this algorithm R-glm. Besides, we have used the somers2 () R function included in the Hmisc package for estimating the AUC. This function computes the c-index explained earlier.

For the new proposed algorithms based on EDAs, we have developed our own implementation in $\mathrm{C}++$. EDAs were run with two different fitness goals: maximizing $\log$ likelihood (UMDA: ${ }_{c}^{G}-\operatorname{logL}$ ) and maximizing the area under the $\mathrm{ROC}$ curve (UMD $\Lambda_{i:}^{G}-\Lambda \mathrm{UC}$ ).

The parameters used to run $\mathrm{JMDA}_{c}^{G}$ were (see lig. 1): (i) population size of 200 individuals ( $M=200)$, (ii) the best. 100 individuals were selected for the learning step ( $N=100$ ), and (iii) the change in the fitness value average between successive gencrations was chosen to assess the convergence of the algorilhms. These parameters were tuned after some extra experiments.

As commented in Sect. 4, a 10-fold cross-validation was used to estimale model performance measures, both in R-glm and $\mathrm{UMDA}_{c}^{G}$ algorithms (this process is shown in Iig. 2 for the EDA-hased algorithms). Due to the stochastic nature of this validation method, each experiment was nun five different times, therefore having a $5 \times 10$-fold cross-validation (Bouckaert and lirank 2004).

\subsection{Comparison of algorithms}

Table 2 summarizes the experimenlal results rellecting the average and standard deviation of the performance measures over all executions carried out with $\mathrm{R}$ (R-glm) arth the two FDA algorithms. Nole that. for cach algorithm we show nol orly the optimized meatsure but also the other measure in order to get an insight into the relattionships between thein. 


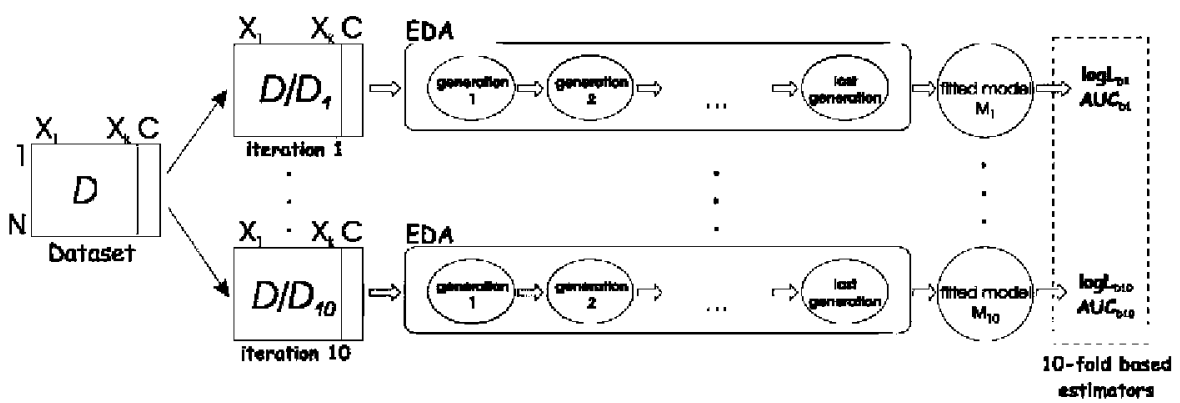

Hig. 2 Assessing the performance of LMUA ${ }_{r}^{G}$ algorithms using 10-fold cross-validation

Tahle 2 Summary of the experimental results reflecting the average and standard deviation of all performance measures over the five exceutions, besides the average computation time for each fold. $\wedge$ means that EDA exhibits a statistically significant better behavior when compared to R-glm (p-value $<0.05$ ). $\nabla$ is for the opposite significance. Filled symbols are used for the performance measure that is being optimized by the associated algorithrt

\begin{tabular}{|c|c|c|c|c|}
\hline \multirow[t]{2}{*}{ Data sel } & \multirow[t]{2}{*}{ Algorithin } & \multicolumn{2}{|c|}{ Model performance meisures } & \multirow[t]{2}{*}{ Compulation time (s) } \\
\hline & & $\log \mathcal{L}$ & $\mathrm{AUC}$ & \\
\hline \multirow[t]{3}{*}{ Breast } & R-glm & $-70.97 \pm 2.34$ & $0.9936 \pm 0.0003$ & 0.24 \\
\hline & $\mathrm{UMDA}_{\mathrm{c}}^{\mathrm{G}}-\log \mathrm{L}$ & $-70.36 \pm 0.84$ & $0.9937 \pm 0.0001$ & 2.80 \\
\hline & $\operatorname{IMDA} A_{c}^{G}-A \mathrm{UC}$ & $-84.70 \pm 6.20 \nabla$ & $0.9930 \pm 0.0015$ & 3.13 \\
\hline \multirow[t]{3}{*}{ Diabetes } & R-glm & $-320.85 \pm 1.60$ & $0.8298 \pm 0.0008$ & 0.32 \\
\hline & $\mathrm{UMDA}_{c}^{G}-$ logL & $-319.48 \pm 1.76$ & $0.8318 \pm 0.0011$ & 4.24 \\
\hline & $\mathrm{UMHA}_{c}^{G}-\mathrm{AUC}$ & $-1526.52 \pm 241.56 \nabla$ & $0.8267 \pm 0.0082$ & 5.48 \\
\hline \multirow[t]{3}{*}{ ICU } & R-glm & $-142.72 \pm 25.70$ & $0.7754 \pm 0.0183$ & 0.49 \\
\hline & I MIMA $G_{c}^{G}-$ logl & $-138.67 \pm 16.79$ & $0.7617 \pm 0.0068$ & 4.66 \\
\hline & 【MPA $G$-ALIC: & $-497.73 \pm 40.19 v$ & $0.7773 \pm 0.0082$ & 9.89 \\
\hline \multirow[t]{3}{*}{ Prostate } & R-glm & $-202.09 \pm 2.18$ & $0.8017 \pm 0.0041$ & 0.20 \\
\hline & $\mathrm{EM}\left[\mathrm{M}_{c}^{\mathrm{G}}-\log \mathrm{L}\right.$ & $-199.25 \pm 1.94$ & $0.8066 \pm 0.0051$ & 1.82 \\
\hline & $\mathrm{UMLA}_{c}^{G}-\mathrm{ALC}$ & $-206,02 \pm 2.60 \nabla$ & $0.8096 \pm 0.0076 \wedge$ & 2.84 \\
\hline \multirow[t]{3}{*}{ UIS } & R-glm & $-320.77 \pm 0.64$ & $0.6220 \pm 0.0030$ & 0.18 \\
\hline & $\mathrm{UMEA}_{C} G-\log \mathrm{L}$ & $-318.98 \pm 0.95 \wedge$ & $0.6307 \pm 0.0037 \Delta$ & 3.99 \\
\hline & $\mathrm{UMDA}_{C}^{G}$-AUC & $-485.15 \pm 29.92 \nabla$ & $0.6417 \pm 0.0106$ & 6.94 \\
\hline \multirow[t]{3}{*}{ Adult-r } & R-glm & $-2832.48 \pm 8.78$ & $0.8449 \pm 0.0015$ & 1.59 \\
\hline & I MIDA & $-2843.57 \pm 5.37$ & $0.8442 \pm 0.0018$ & 218.37 \\
\hline & $\mathrm{UMLA}_{c}^{G}-\Lambda \mathrm{LIC}$ & $-12821.53 \pm 418.47 \nabla$ & $0.8447 \pm 0.0012$ & 371.59 \\
\hline
\end{tabular}

The Mann-Whitney test was used to compute the statistical significance of the difference between the algorilhms. $\Lambda \Delta$ symbol (a $\nabla$ symbol) in a value means that the corresponding LDA algorithm reveals a statistically significant better (worse) behavior than R-glin with a $p$-value $<0.05$. Filled symbols are used for the performante measure that is being optimized by the associated algorithm. The absence of the sym- 
bol means that there was not enough evidence to reject the null hypothesis of equal behavior of the algorithms.

Several conclusions can be extracted from lable 2 with respect to the algorithms:

- The UMD $\Lambda_{c}^{G}$ - $\log \mathrm{I}$, algorithm achicves at. least. the same resuls as the R-glm a]gorithm. Most of the time the dillerences are negligible. In lact, there is only a statistically significant difference in the $\log \mathcal{L}$ value for the UIS data set in favor of the EDA ( $p=0.01$ ), see the second row of each data set, under fhe $\log C$. column. In conlrast, R-glm is never stalistically superior to the FDA. Note that. ith this case both algorithms are using the same fitness function, $\log \mathcal{L}$, but a completely different search strategy.

While oplimizing $\log C$, we can record the corresponding $\triangle U C$. (socond row, last column). In this case, $\Lambda U C$ as a complementary measure, exhibils the same behavior when the search is carried out using R-gIm and LDA algorithms. There is one statistically sionificant difference in the AUC value for the UIS data set in favor of the FDA $(p=0.007)$.

- The behavior of the UMDA ${ }_{c}^{G}$-AIJC algorithm is now analyzed. Here we compare the AUC outputted by UMDA $c$-AUC algorithm, which is its objective function, and the $A U C$ conesponding wilh the model that. R-glm finds (see the thitd row of each (ala set, under the AUC column). For Breast, Diabetes, ICU and $A d u l t-r$ data sets, the differences are negligible. However, for Prostate $(p=0.05)$ atnd UTS $(p=0.007)$ data sets, EDA is again statistically superior to R-glm.

When we look at the $\log \mathcal{L}$ values, recorded in our LDA algorithm as a complementary measure not used for the optimization (third row of each data set, under the $\log C$ column), R-gim always displays statistically signilicant dilferences versus EDA ( $\mu=0.007$ always except for the Prostate data set, with $p=0.03$ ).

Therefore, utder the same optimization problem of maximizing $\log \mathcal{L}$, our $\mathrm{ED} \wedge$ (UMDA $c^{G}-\log L$ ) algorithm behaves as a strong competitor of the R-glm algorithm, achieving similar and sometimes better results.

On the contrary. when we compare a cerlain measure yiclded by algorilhms that optimize different objectives, the results use to favor the algorithm that optimizes the measure. Thus, if we compare the $\log C$ values outputted by the UMDA $c$-AUC algorithm and the R-glm algorithm, the latter algorithm is better. Ilowever, when we compare the $\Lambda \mathrm{LC}$. values outpulted by the $U \mathrm{MT} \Lambda_{c}^{G}-\Lambda \mathrm{UC}$ algorithm and the R-glm algorithm, both algorithms almost tie, with the LDA algorithm being slightly superior.

In lerms of compulational lime, EDA-based algorilhms are slower than R-glm, but required times are quite reasonable. lor the first five data sets, while R-glm ranges between $0.18 \mathrm{~s}$ and $0.49 \mathrm{~s}$, UMLA ${ }_{c}^{G}$-logL ranges between $1.82 \mathrm{~s}$ and $4.66 \mathrm{~s}$ and UMDA $_{c}^{G}$-AUC ranges between $2.84 \mathrm{~s}$ and $9.89 \mathrm{~s}$. For the Adult $-\mathrm{r}$ data set, with

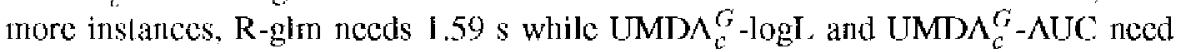
$218.37 \mathrm{~s}$ and $371.59 \mathrm{~s}$, respectively (see Table 2 ).

Note that optimizing an objective does not guarantee a good behavior of other possible objeclives. How calibration and discriminalion measures are related in logistic regression models is an aim in this paper and will be analyzed in the next subsections. 


\subsection{Joint evolution of performance measures}

The results also show that optimizations wilh $\log L$ as filness luthclion achicve good results in all performance measures while optimizations with AUC as fitness function achieve only good results in AUC values. Just another point of view: optimizing calibration results in optimizing discrimination, but optimizing discrimination does not result in oplimizing calibration. As discussed in Secl. 3, $\log L$ is a calibralion measure while $\Lambda U C$ is a discriminalion measurc.

It is possible to verify this situation by analyzing the evolution of the performance measures during the optimizations of the different fitness functions. Itor the Diabetes data set, the evolution of each measure. AUC and $\log C$, during the maximization of $\log C$ can be seen in Fig. 3. Similarly, the evolution during the maximizalion of AUC in the Diabetes data sel can be found in Fig. 4. The hehavior on the olher live data sets is analogous.

Note that each logistic regression model, that is, specific values for the parameters $\beta_{0}, \beta_{1}, \ldots, \beta_{k}$, has associated AUC and $\log \mathcal{C}$. values represented in the curves of the figures. Sinee we have already compared the algorithms, from now on the models will be filled hy using the whole data set, i.c., by resubstitution.

As shown in Fig. 4 for the Diabetes data set during AUC maximizalion, when AUC values become stable (generation 12 ) at approximately $0.839, \log C$ values range from -3064 to -1073 . However, as observed in lig. 3 , during $\log \mathcal{L}$ maxjmization, when $\log \mathcal{L}$. values become stable (generation 18 ) at approximately -366 , AUC also reatches good and stable values. This means that log $\mathcal{C}$ optimization leads to AUC oplinization bul nol vice versa.

Moreover, the final $\log L$. values when maximizing AUC are different depending on the execution. For example (see Table 2), in the Diabetes data set with stable filal $\Lambda \mathrm{UC}$, values (mean 0.8267 wilh standard deviation 0.0082 ) there is a high

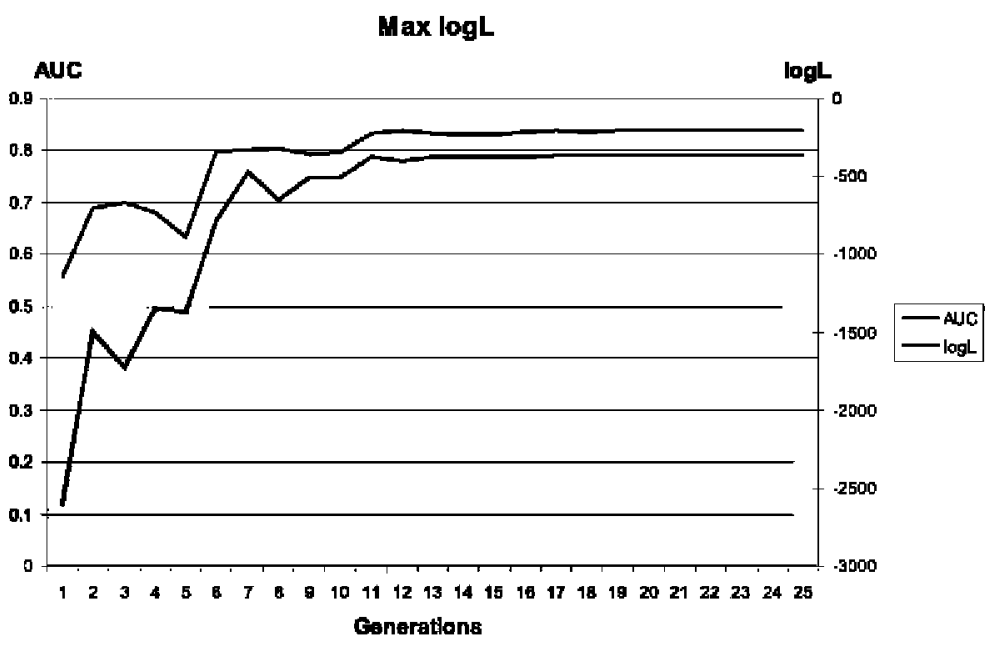

Fig. 3 Evolution or $\log \mathcal{C}$ and $\mathrm{AUC}$ in the UMDA ${ }_{\mathrm{C}}^{G}-\log _{\mathrm{b}} \mathrm{L}$ algorithm for the Diabetes dala set 


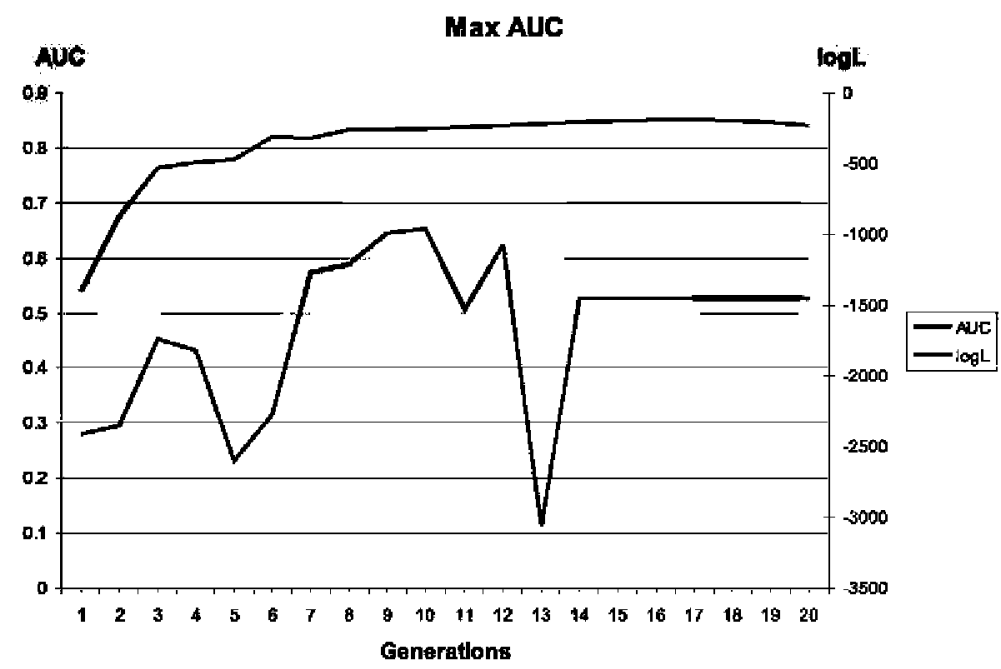

Fig. 4 Evolution of $\log L$ and $A U C$ in the UMD $\Lambda_{c}^{G}-A U C$ algorithm for the Di abotes data set

Table 3 UMDA C-AUC algorithm rinal results of the fiwe exccutions for Diabetes

\begin{tabular}{lll}
\hline Execution & Final $\log \mathcal{C}$ & Final ALC \\
\hline 1 & -1440.478 & 0.8400 \\
2 & -1413.735 & 0.8397 \\
3 & -1395.572 & 0.8334 \\
4 & -1096.662 & 0.8398 \\
5 & -1407.625 & 0.8332 \\
\hline
\end{tabular}

variance in the final $\log \mathcal{L}$ values (mean -1526.52 with standard deviation 241.56) because each of the executions reaches quite different search space points.

Table 3 reports the final $\log \mathcal{L}$ and $\mathrm{AUC}$ values of the Ingistic regression models that have been achieved in the five executions of the UMDA ${ }_{c}^{G}$-AUC algorithm for the Díabetes data set.

An explanation for this lies in the different shapes of the optimization functions. The $\log \mathcal{L}$ is a concave function of the $\beta$ coefficient, and the free variation of $\beta$ in a convex set guarantees that there are no local maxima on the log-likelihood surface of a logistic regression model. 'That implies an easy-to-reach optimization, which also brings incidentally good values in other performance measures like AlC, as we have showll.

Nonetheless, the AUC surface has many local maxima, each one with similar AUC values but different $\log \mathcal{L}$ values. This implies a harder optimization prohlem, with the added difficulty of having to be cautious with the (perhaps bad) $\log \mathcal{C}$ value associated to a good AUC value obtained when maximizing the AUC function.

This is also observed in I'ig. 5 that depicts the search space points that the LDDA algorithms visited in the whole optimization process of the Diabetes data set. lior 


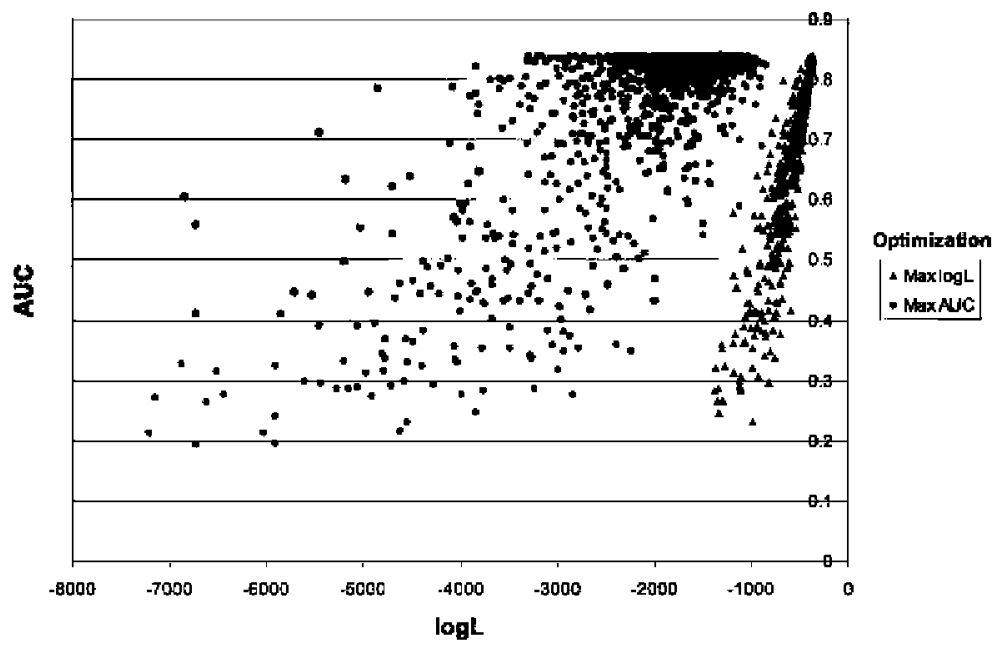

lig. 5 Performance measures, $\log \mathcal{L}$ vs. ALC., of all visited logistic regression models during the two EDA-based optimization processes of the Diabetes data set

cach visited point the performance estimates of $\log \mathcal{L}$ and $\Lambda U C$ are plotled. The behavior on the other five data sets is quite similar.

\subsection{Pareto front in the calibration vs. discrimination space}

Our previous experiments with EDAs have shown that optimizing log $\mathcal{L}$ achieves good results in AUC. This provides the basis to develop an ad hoc method to explore promising regions of the bi-objective space of calibration ( $\log L$ ) vs. discriminalion (Al)C). The method proceeds as follows. The LDA algorithm is run many times, each one with the same value of parameter $\mu_{i 0}$ and different $\sigma_{i 0}(i=0,1, \ldots, k)$, both determining the normal density function of the $\mathrm{JMDA}_{c}^{G}$ model at the initialization step, see Sect. 5.2. Parameter $\mu_{i 0}$ is fixed as the component $i$ of the solution achieved by the Newlot-Raphson method. Parameler $\sigma_{i 0}$ is progressively increased lo collarge the space to be explored. Since $\log \mathcal{C}$ is the objective function for the EDA, this naive procedure leads to points wilh hopefully good values not only in $\log L$, bul irl AUC.

In the bi-objective space, non-dominated points are of interest as optimal solutions in the sense of trol having any other point. that is equal or better with respect to all the objective functions. The resulting set is the non-dominated or efficient set, also called Pareto front (Stener 1986). Circles (magenta color) of Fig. 6 show the approximate Pareto front obtained by the ad loc method described above.

Alternative ways to find the non-dominated points would consist of undertaking bi-objeclive oplimizalions. $\Lambda$ recenl. rescarch by 7 hang a al. (2008) proposes a regularity model based multiobjective LDA (RM-MLDA) for continuous multiobjective optimization problems. Inder mild smoothness conditions, il holds that the Pareto set is a piecewise continuous manifold of dimension $r-1$, where $r$ is the number of objectives. The idea is to exploit explicitly this regularity property of the Pareto set in huilding the probabilistic model the LDA needs. LDA constructs the probability 

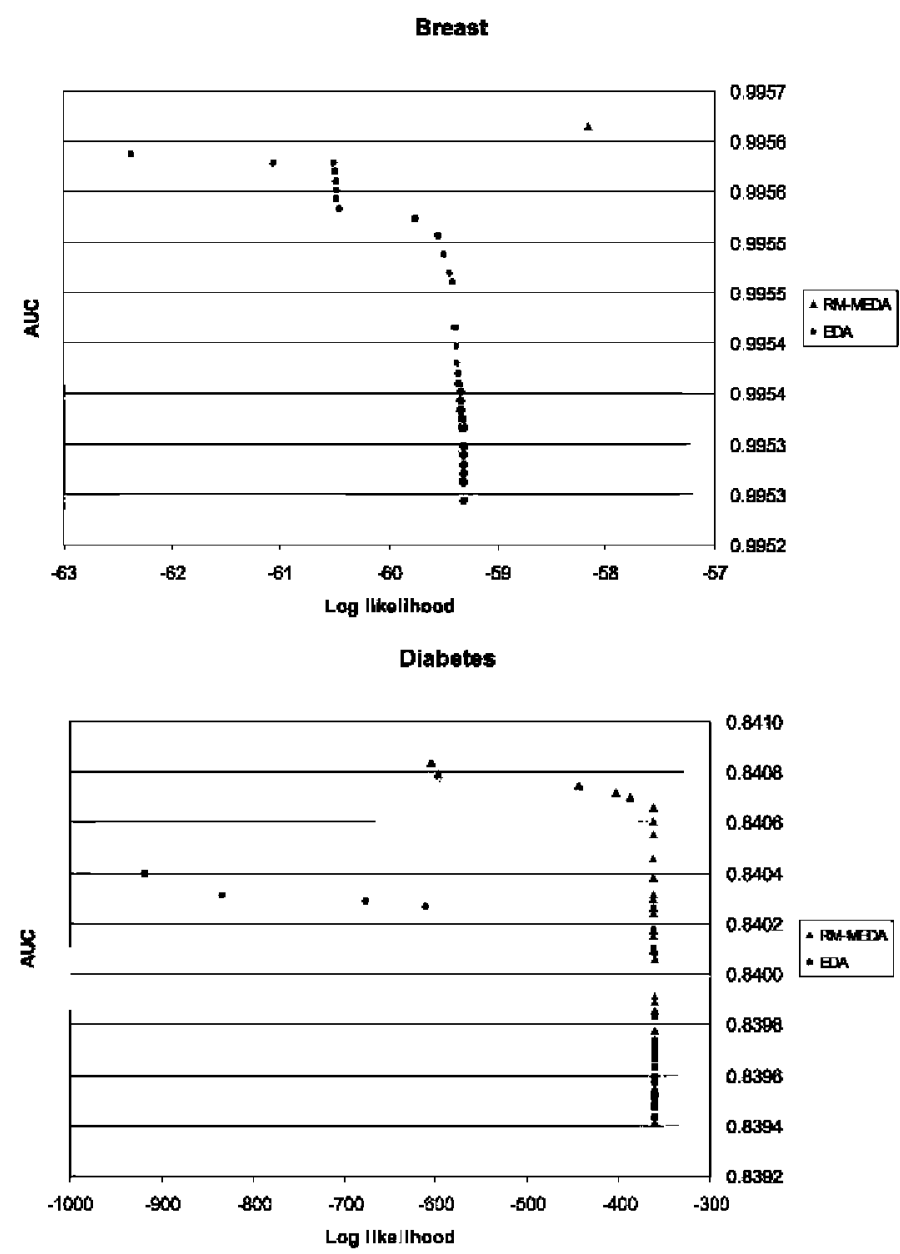

Fig. 6 (Color online) Pareto fronts found by the ad hoc provedure (magena cincles) and by RM-MEDA (blue triangles) of all diklis sels

model whose centroid is a piecewise continuous manifold, via a local PCA algorithm. Experimental results show a glohal superiority of RM-MLDA against recent competitive multiobjective melahcuristics, includitgs NSGA-II (T)b el al. 2006). Triangles (blue color) of Fig. 6 show the approximate Pareto front obtained by RM-MEDA.

It is remarkahle that for Breast, the Pareto front includes an isolated point (locatcd at the right upper comer). This point is only found by RM-MFDA. The belavior of RM-MEDA is also better for the rest of data sets as compared with our ad hoc method, specially as regards their AUC values. For ICU and AduIt-r, all points found by our procedure are dominated by some point oblained by RM-META. However, for Diabetes, Frostate and UIS, both methods find a similar number of points that are non-dominated between them. The chosen scale of lig. 6 , in particu- 

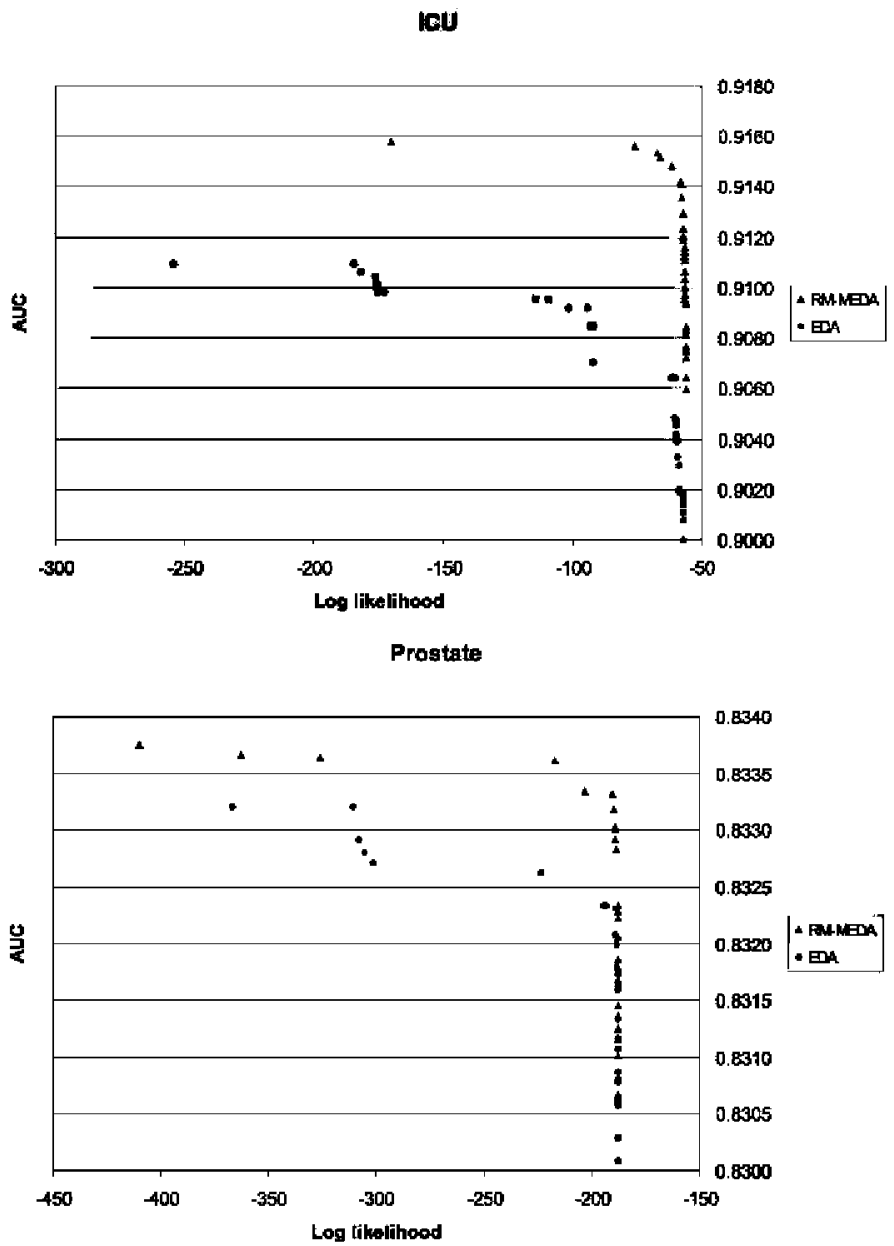

Fig. 6 (Continued)

lar for the $\log \mathcal{L}$, avoids clearly visualizing this fact, since we tried to empliasize the dilferences between the $\triangle \mathrm{UUC}$. objective values for the two methods.

\section{Discussion on benefits of the EDA approach}

The advantages of using our EDA framework rather than the traditional numerical methods may be enumerated as follows:

- EDA is fexible cnough to cope with any oplimization funclion. EDA does not require derivative information nor matrix inversions. EDA does not need a function with an explicit formula, which is the case of the AUC. Function, On the conlrary, numerical methods are only designed for optimizing the $\log \mathcal{C}$. function demanding matrix inversions. 

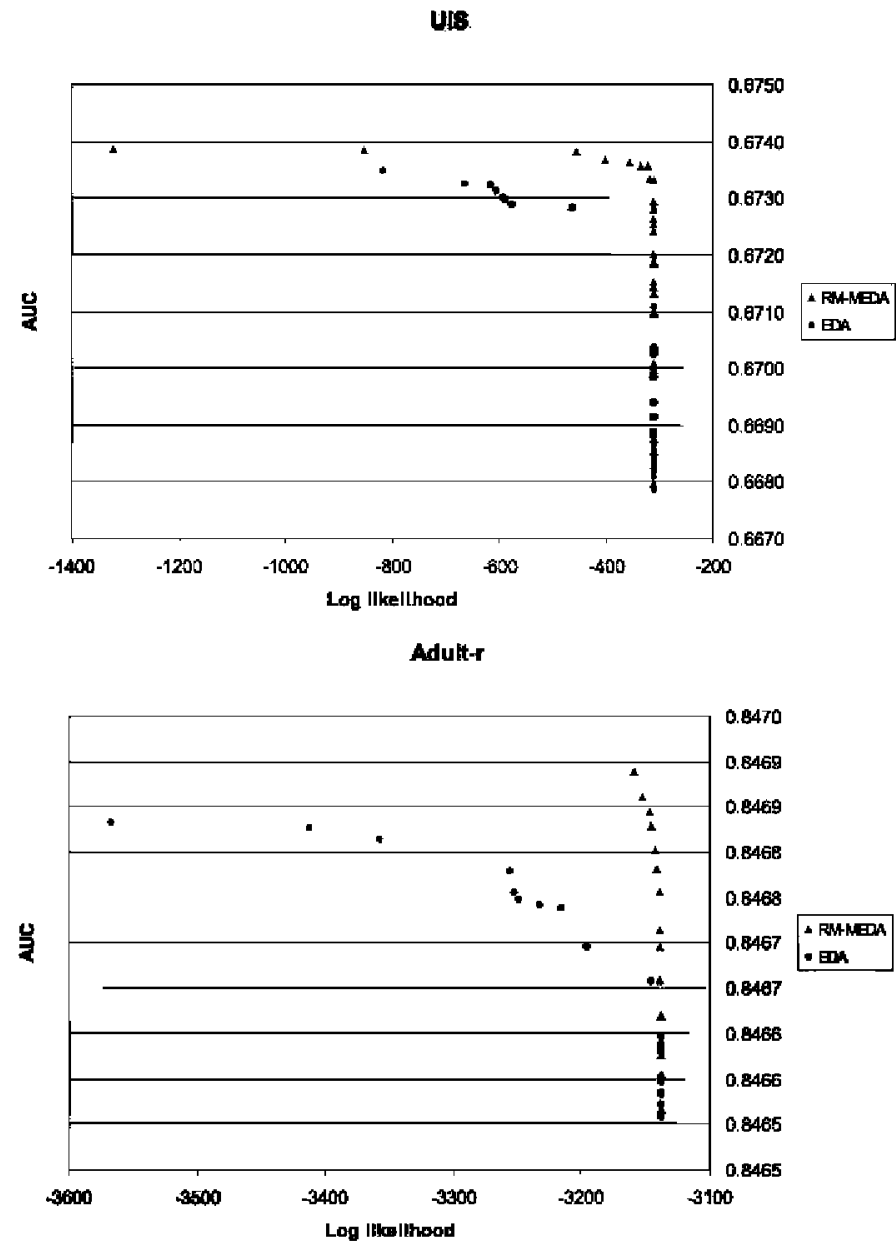

Fig. 6 (Conimuted)

- LDA may use any performance measure. We have used $\log \mathcal{L}$ and AUC but other performance measures may be used. In fact, we tried with the IIosmer-Lemeshow statistic calibration measure (Hosmer and I emeshow 2000). The resuls were not shown, since they produced very high correlations (greater than 0.90 ) with the $\log$ likelihood function. We tlso tried with the Brier score (Brier 1950), with worse results lhan the current oncs.

- LDA is a parallel and an inherently global optimal search technique that simultaneously eviluates many points in the parameter space and is more likely to converge loward the global solution of the optimizalion problem. Thus, il avoids being trapped at local optima. We are aware of local optima with the AUC function but not with $\log C$, which is concave.

- Morcover, numerical procedures like Newion-Raphson usually converge, bul overshooting can occur (McLachlan 1992). Also, they exhibit some dependence on the 
initial starting conditions for convergence to be guaranteed, although we do not have experimented this perhaps due to the relined implementation of the R program or due to the chosen data sets. Being a population-hased search method, the EDA approach is unlikely to suffer from these drawbacks.

- EDA is nol in fluenced by situalions when the number of covariates is relatively large compared to the number of observations. Traditional numerical methods do not work in this scenario, having problems in estimating parameters properly.

- EDAs create a framework where we can study calibralion and discrimintition measures. We could investigate the behavior of LDAs when maximizing calibration or discrimination as compared to R-glm, with the only disadvantage of having bad $\log \mathcal{L}$ values when the $A U C$ is optimized. The joinl. evolution of both measures has been analyzed, where a constructive interaction between calibration and discrimination was found when optimizing calibration, and more independence between both was lound when optimizing discrimination.

- l'urthermore, in the space of the two objectives of calibration vs. discrimination, the Pareto front was found with a competitive and sophisticated multiobjective EDA, RM-MEDA. In contrast, a simple uni-objective EDA procedure guided by the $\log \mathcal{C}$ yielded a slightly worse approximation to the Pareto front.

These last two benefits of EDAs are not obtained with traditional numerical methods which only search for the point. Ihat maximizes the log-likelithool.

On the other hand, metaheuristics such as GAs, tabu search, ant colony, scatter search, elc., hold all the propertics above. However, FDAs sland oul against olher metaheuristics and traditional numerical methods because of the following characteristics:

- EDAs avoid the tuning of the values of many parameters.

- EDAs caplure explicilly the probabilistic dependencies among paramelers $\beta_{i}$ 's what is a useful information in Iogistic regression models. Different graphical structures may show chains, trees, polytrees, and general acyclic structures.

Some distdvantages follow:

- In a way the stochastic nature of EDA algorithms may be considered a disadvantage, since different executions may lead to slightly different results.

- All this comes at the price of having a higher compulational cost, which in our case was alleviated by the powerful machines available to us (see Acknowledgments).

\section{Conclusions and future research}

To our knowledge, this was the first description of utilizing LDAs to estimate regression parameters, as well as the first one to compare different optimization functions, usittg maximum log-liketihood and the Newton-Raphson mellod irnplentented ito $R$ as a benchmark. Although our results did not differ dramatically from those of the benchmark, it is important to emphasize some points. First. MLE estimation requires the inversion of a malrix and will simply nol work if the number of variables exceeds the number of observations. This is often the case in contemporary data sets. 
Although dimensionality reduction and feature selection are active areas of research and may help remedy this situation, the use of LDAs can offer an attractive alternative, as the aloorithms do not offer this limitation. Furthermore, we have only utilized a very simple form of LDAA, and it is expected that more complex forms will yield beller results. Fitlally, our intitial exploration of alternative oplimization [unctions suggests that it may be better to favor functions that take into account calibration more strongly than discrimination, and provides initial empirical support for the use of these functions.

Our results support the need for lurther exploration of how to better estimale parameters for logistic regression. IIowever, the use of EDAs is by no means limited to this type of models and further exploration in terms of their use in the context of more complex models is also warranted.

\section{References}

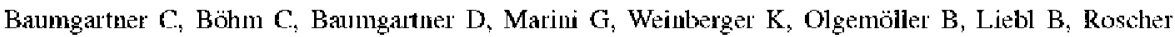
A (2004) Sepervised machine learning techniques for the classification of metabolic disorders in newbollus. Bioinfolmatics 20(17):2985-2996

Blanco $\mathrm{K}$, Inza I, I arranaga $\mathrm{P}$ (2003) I.earning Bayesian networks in the space of stractures by estimation of distrihution algorthms. Int I Intcll Syst 18:205-220

Bouckact $\mathrm{k}$, Frank E (2004) Fvaluating the replicability of significance tests for comparing learning

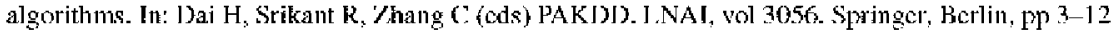

Bradley AP (1997) The use of the area under the ROC curve in the evaluation of machine learning alsorithtm. Pattcrit Recogn 30(T):1 I45-1159

Brier G (1950) Verifration of forecusts expressed in terms of probabilities. Monthly Weaher Rev 78:1-3

Deb K. Sinhi A. Kukkonen S (2006) Multi-objeclive test problems, linkages, and evolutionary methodologies. In: GECCO-2006, Genelic and evolutionary compulation con[erence, vo] 2. ACM Press. New York, pp I 141-1148

Fawcell T (2003) ROC graphs: Noles and prawical considerations for data mining researchers. Technical reporl, IIPL 2003-4, IIP Labs

Goldbers DE (1989) Genelic algorithms in seareh, optimizalion, and machine learning. Addison-Wesley, Resding

Hajek J, Zidak ZB, Sen PK (1999) Theory of rank tests, 2nd edh. Academuc Press, San Diego

Hanley $\mathrm{J} \Lambda$, McNeil BJ (1982) The meaning and use of the area under a receiver operating characteristic (ROC) curve. Radiology 143:29-36

Harrell FE. Lee KL. Califf R. Pryor D, Rosati R (1984) Regression modelling strategies for improved prognostis prediction. Stat Med 3:143-152

Harrell HF. I se KI. Mark DB (I996) Multivariable progmostic models: 1ssues in deweloping models, evaluating assumptions and adequacy. and measuring and reducing errors. Stat Med 15:361-387

Hilden $I$ (1991) ' the area under the ROC Curve and its competitors Med Decis Mak 11(2):95-10]

Hotton N., Browit HR, Qian L. (2004) lise of $\mathrm{R}$ as a toolbox for mathematical statistics cxplotation. Am Stat $58(4): 343-357$

I Losner DW, Lemeshow S (2000) Applied logislic regression, 2nd edn. Wiley, New York

Ihaka R, Gentleman R (1996) R: A languate for data analysis and graphics. J Compua Graph Stat 5:229314 
Inza I, Lalraltaga P. Exeberria R, Sierla B (2000) Feature subset selestion by Bayesian network-based oplimization. J Artif Intell Res 123(1-2):157-184

Kiang MY (2003) A comparative assessment of classilicalion methods. Decis Support Syst 35:441-454

Larrañaga $P$, Lozano EA (2002) Estimation of distribution algorithms. A new lool for evolutionary computation. Kluws Academic, Jordrecht

J.artañaga $\mathrm{P}$, Htxehertia $\mathrm{R}$, I orano JA, Peña JM (2000) Optimization in entinuous domains by karning and simulation of Gaussian networks. In: Workshop in optimization by bailding and using probabilistic models within the 2000 genetic and evolutionaly computation conference, GECCO 2000 , pp $201-204$

Lasko TA. Bhatg wat JG. Zot KII, Ohno-Machato L (2005) The use of ROC curves in biomedical informatics. J Biomed Inform 38:404-415

J ozano .A, L arrañaga P. [nza I. Bengoctxea $E$ (2006) Towards a new crolutionary computation. Advances in estimation of distribution algorithms. Springer, New York

McLachlan G (1992) Discrimimant analysis and statistical pattern recognition. Wiley, New York

Miıka T (2003) $A$ comparison of numerical optimizers for logistic reglession. Technical report. 758 , Carnegie Mellon Universily

Nakamichi R. Imoto S. Miyno S (2004) Case-control study of binary disease trail considering jnlelaclions hetween SNPs and environmental effects using logistic regression. In: Fourth IEEE symposium on hiointormatics and hiocngincering, vol 21 , pp $73-78$

Newnan [D, Hettich S, Blake C. Merz C (1998) LICI repository of machine learning databases

Ng $A$, Jordan M (2001) On discrimimative versus generative classifiers: A comparison of logistic regression and nave Bayes. In: Proceedings of NIPS, vol 14, Pp $841-848$

Pepe MS (2003) The stitistical evaluation of medical tests for chassilicalion and prediction. Oxford Liniversily Press, Oxfort

Prowost F. Frawcell $T$, Kohivi $R$ (1998) The case against atcuraty estimation for comparing induction algorithms. In: Procedings 15 th international conference on machine leaming. Morgan Kaufmann. San Matco, pp 445-453

R Development Gore Team (2004). R: A language and environment for statistical computing. R Foundation for Statistical Computing, Vieuna, Austila. ISBN 3-900051-07-0

Romero T: Larrañaga P. Sierra B (2004) Learning Bayesian networks in the space of orderings with estimation of đistribution algorithms. Int I Putlern Recogn Artif Intell 4(18):607-625

Ryan IP (1997) Modern regression methods. Wiley. New York

Steter RE (1986) Multiple criteria oplinization: Theory, computation, and application. Wiley, New York

Steyerberæ L, Borsboom G, vau Ilouwelingen II, Enjkemans M. Ilabbema J (2004) Validation and updating of predictive Iogistic regression models: a study on sample size and shrinkagc. Stat Mcd 23(10):25672586

Stone M (1974) Cross-validatory choice and assessment of statistical predictions. J R Stat Soc Ser B 36:111-147

Thisted RA (1988) Elements of stalistical computing. Chapman and IIall. London

van den E Iotl WB (2003) The area under an ROC curve with limiled information. Med Decis Mak 23:160166

Vinterbo S, Ohto-Machado I. (1994a) A gentic algorithm to sclect variables in logistic regression: Fxample in the domain of myocardial infarctio. J Am Med Inform Assoc 6:984-988

Vinterbo S, Ohno-Machado L (1999b). A recalibration method for predictive models with dichotomous outcomes. In: Predictive models in medicine: Some methods for construction and adaptation. PhD thesis. Norwegith University of Science and Technolony

Winker P. Gilli M (2004) Applications of oplimizalion heuristics to estimation and modelling problems. Computat Stat Data Anal 47:211-223

Thang Q; Thou A, Jit Y (2008) l MM-MHDA: A regularity model basod multiolicetive cstimation of distrilution algorithms. IFEK Trans Hool Comput 12(1):41-63 